Das Vorhandensein von anderen Salzen ausser dem vorliegenden $\alpha$-Methyl- $\beta$-oxybuttersaurem Kalium wurde nicht nachgewiesen. Wurde das Salz mit Schwefelsäure gemäss der Angabe von Miller destilliert, so wurde auch hier das Auftreten von Tiglinsäure, die durch den Schmelzpunkt charakterisiert wurde, dargethan.

Das Vorhandensein von Tiglinsäure in den Oxydationsprodakten des Jalapins mit Barythydrat ist demnach auf die Bildung von genannter Oxysäure, die durch blosses Erhitzen sowohl, als auch mit verdünnter Schwefelsäure Wasser abspaltet, und die zugehörige ungesättigte Säure liefert, zurückzuführen:

$\mathrm{CH}^{8} \cdot \mathrm{CH}(\mathrm{OH}) \cdot \mathrm{CH}\left(\mathrm{CH}^{8}\right) \mathrm{COOH}=\mathrm{H}^{2} \mathrm{O}+\mathrm{CH}^{8} \cdot \mathrm{CH}=\mathrm{C}\left(\mathrm{CH}^{8}\right) \mathrm{COOH}$.

Das Vorkommen von $\alpha$-Methyl- $\beta$-Oxybuttersäure muss aber das Auftreten von Methyläthylessigsäure $\mathrm{CH}^{8} \cdot \mathrm{CH}^{2} \cdot \mathrm{CH}\left(\mathrm{CH}^{8}\right) \mathrm{COOH}$ bei der Zersetzung des Jalapins zum Ausgangspunkt haben.

Die Reihenfolge der Entstehung der isolierten Körper würde demnach von einem solchen Gesichtspunkte aus, Nachstehende sein:

$\mathrm{CH}^{8} \cdot \mathrm{CH}^{2} \cdot \mathrm{CH}\left(\mathrm{CH}^{8}\right) \mathrm{COOH}$ wird durch Verseifen des Jalapins mit Barythydrat abgeschieden; durch überschüssig angewandtes Hydrat entsteht gleichzeitig: $\mathrm{CH}^{8} \cdot \mathrm{CH}(\mathrm{OH}) \mathrm{CH}\left(\mathrm{CH}^{8}\right) \mathrm{COOH}$ die durch Erhitzen in $\mathrm{H}^{2} \mathrm{O}$ und $\mathrm{CH}^{3} \cdot \mathrm{CH}=\mathrm{C}\left(\mathrm{CH}^{8}\right) \mathrm{COOH}$ zerfällt.

Den Untersuchungen von Miller ${ }^{1}$ ) nach ist die Methyläthylessigsäure direkt hydroxylierbar und liefert die zugehörige Oxysäure, welche wiederum in Methylcrotonsäure und Wasser zerfällt.

\title{
Ueber die Essigsäureester des Jalapins und der Jalapinsäure.
}

Von N. Kromer.

(Eingegangen den 15. VI. 1901.)

Die Thatsachen, welche zur Zeit bei der Untersuchung des Jalapins ermittelt sind, erlauben die Voraussetzung, dasselbe als den d-Methyläthylessigsäureester der Jalapinsäure zu bezeichnen und in letzterer Säure eine Glukosido-Jalapinolsäure präformirt zu erblicken. Das Jalapin wäre demnach d-Methyläthylacetyl-Glukosido-Jalapinolsäureester. Sollte dieser Schluss der Wirklichkeit entsprechen, so müsste die in Wasser lösliche Glukosido-Jalapinolsäure = Jalapinsäure

1) Liebig's Annalen Bd. 200, pag. 285. 
durch Ueberführung in die entsprechenden Säureester unlöslich werden, amorph sein, und "Harznatur" annehmen. Andererseits aber musste es von Interesse sein zu erfahren, in wie weit das Jalapin dem Eintritt von Säuregruppen zugänglich ist. $\mathrm{Zu}$ diesen Versuchen wurden die betreffenden Substanzen mit der dreifachen Menge Essigsăureanhydrid und der gleichen Menge entwässerten Natriumacetats im Einschlussrohre 5 Stunden lang auf $135^{\circ}$ erhitzt und der Röhreninhalt auf dem Wasserbade bis zum völligen Entweichen des tüberschüssig angewandten Anhydrides in einer Porzellanschale abgedampft. Die betreffenden Rückstände wurden hierauf mit Aether ausgezogen und die Aetherlösung mit Wasser gewaschen. Die nach dem Entfernen des Lösungsmittels und dem voraufgehenden Entwässern der Lösung durch Chlorcalcium hinterbliebenen Rtickstände, welche, wie es bemerkt sei, durchweg amorph waren, wurden zuerst anf dem Wasserbade und alsdann bei $80-100^{\circ}$ getrocknet. Um dieselben zu verreiben wurden die Rückstände, welche bei dieser Operation stark elektriach wurden, mit niedrig siedendem neutral reagierenden Petrolather angefeuchtet. Das Jalapin war auf gewöhnliche Weise vorher durch Wasser und Petroläther gereinigt und die angewandte Jalapinsäure durch absoluten Aether gefallt worden.

Bevor zur Untersuchung der genannten Ester geschritten wurde, musste die Menge Alkalihydrat ermittelt werden, welche das Jalapin zu seiner Verseifung erforderte.

1. $1,7635 \mathrm{~g}$ Jalapin erforderten in Alkohol gelöst zur Rotfärbung bei Gegenwart von Phenolphtalein $0,4 \mathrm{ccm}$ KHO $(1 \mathrm{ccm}$ KHO $=0,0485495 \mathrm{~g}$ $\mathrm{KHO})=1,10 \% \mathrm{KHO}$. Hierauf wurden $20 \mathrm{ccm}$ derselben Lauge zugegeben und 1 Stunde lang am Rückflusskühler gekocht, wobei $12,1 \mathrm{ccm}$ KHO zurücktitriert wurden $\left(7,5 \mathrm{ccm} \mathrm{KHO}=24,4 \mathrm{ccm} \mathrm{H}^{2} \mathrm{SO}^{4}\right.$; verbraucht warden $39,3 \mathrm{ccm}$ H'SO'-Lösung). Mithin waren 7,9 ccm KHO $=0,38354105 \mathrm{~g}$ KHO $=21,74 \%$ KHO zur Verseifung erforderlich gewesen.

2. $1,2283 \mathrm{~g}$ Jalapin warden in vorher beschriebener Weise behandelt und verbrauchten $0,3 \mathrm{ccm}$ KHO zur Rötung $=1,20 \%$ KHO; zur Verseifung waren $5,4 \mathrm{ccm}$ KHO $=21,63 \%$ erforderlich.

Das Acetyl-Jalapin war gelb gefärbt, amorph und teilte hinsichtlich der physikalischen Eigenschaften und seiner Löslichkeit die Eigenschaften des Jalapins, war jedoch spröder als dieses.

1. 2,4855 g Acetyl-Jalapin erforderten zur Aufhebung der Sauerung $0,6 \mathrm{ccm}$ alkoholische $\mathrm{KHO}=1,17 \% \mathrm{KHO} ; 22,2 \mathrm{ccm} \mathrm{KHO}(1 \mathrm{ccm}=0,0485495 \mathrm{~g}$ KHO) wurden zur Verseifung verbraucht $=43,36 \% \mathrm{KHO}$.

2. $0,8677 \mathrm{~g}$ derselben Verbindung erforderten zur Spaltung $8 \mathrm{ccm}$ alkoholische Kalilauge $(1 \mathrm{ccm}=0,0492056 \mathrm{~g} \mathrm{KHO})=45,36 \% \mathrm{KHO}$.

Arah. d. Pharm. cCXXXIX. Bds. 5. Heft. 
Gefunden KHO in Prozenten für das

Jalapin:

1.2 . 2.

$1,10 \quad 1,20$

Zur Neutralisation:

Zar Verseifung des Esters: $21,74 \quad 21,63$

Mittel: 21,685 .
Acetyl-Jalapin:

1. 2.

$1,17-$

$43,36 \quad 45,36$

44,36 .

Dieser Mehrverbrauch des Acetyl-Jalapins von 22,675\% KHO ist dem Eintritte von Acetylgruppen zuzuschreiben, welche 17,37\% $\mathrm{CH}^{3} \mathrm{CO}$-Gruppen entsprechen.

Der Acetylester der Jalapinsäure ist zum Unterschiede von seiner Muttersubstanz in Wasser unlosslich, amorph, hellgelb gefärbt, lässt sich in seidenglänzende Fäden ausziehen und zeigt in seiner ausseren Beschaffenheit vôllige Analogie mit den Convolvulaceenharzen. Ein scharfer Schmelzpunkt konnte auch hier nicht beobachtet werden. Bei $105^{\circ}$ sinterte die Substanz zusammen und wurde bei $120-125^{\circ}$ zähfliusssiger.

0,2832 g Substanz gaben nach dem Trocknen im Exsiccator beim Verbrennen:

$0,175 \mathrm{~g} \mathrm{H}^{2} \mathrm{O}$ und $0,5535 \mathrm{~g} \mathrm{CO}^{2}$ $6,92 \% \mathrm{H}-53,30 \% \mathrm{C}$.

1. $0,6614 \mathrm{~g}$ des Acetylesters erforderten in Alkohollösung zur Neutralisation der freien Säure $=0,4 \mathrm{ccm}$ KHO $(1 \mathrm{ccm}=0,0485495 \mathrm{~g} \mathrm{KHO})$ $=2,93 \%$ KHO. Zur Verseifung wurden 6,5 ccm KHO $=47,8 \%$ KHO gebunden.

2. $1,1113 \mathrm{~g}$ des Esters einer andern Darstellung verbrauchten zur Sättigung 0,6 ccm KHO-Lösung $(1 \mathrm{ccm}=0,0492056 \mathrm{~g} \mathrm{KHO})=2,76 \% \mathrm{KHO}$. Zur Spaltung des Esters waren 11,4 ccm KHO $=50,47 \%$ KHO erforderlich.

3. $0,711 \mathrm{~g}$ derselben Substanz erforderten zur Neutralisation $0,4 \mathrm{ccm}$ KHO obiger Konzentration $=2,76 \%$ und zur Verseifung $7,3 \mathrm{ccm}$ KHO $=$ $50,52 \%$ KHO.

4. $0,5668 \mathrm{~g}$ der zu den Versuchen benutzten, durch Aether gefältten Jalapinsăure verbrauchten bei der Titration unter Anwendung von Phenolphtalein als Indikator $0,4 \mathrm{ccm}$ alkoholischer $\mathrm{KHO}=3,47 \% \mathrm{KHO}$.

Das Jalapin, die Ester desselben und die der Jalapinsäure werden aus ihren Lösungen in wasserfreien Alkohol durch alkoholische Kalilauge nach kurzer Zeit in amorphen Flocken ausgeschieden.

Durch mässigen Wasserzusatz werden die Ausscheidungen in Lösung gebracht. Es hat den Anschein, als ob hierbei eine Verbindung der genannten Körper mit Kalihydrat eintritt, die durch Wasser zerstört wird.

Zur direkten Bestimmung der eingetretenen Acetylgruppen wurde der Acetylester mit $\mathrm{KHO}$ in Alkohollösung verseift und der von Alkohol befreite Rückstand mit sirupförmiger Phosphorsäure solange destilliert, bis das übergehende Destillat keine saure Reaktion mehr aufwies. 
$0,883 \mathrm{~g}$ der Substanz, welche im Exsiccator getrocknet war, ergaben ein Destillat, das $18,8 \mathrm{ccm} \mathrm{NaOH}(1 \mathrm{ccm}=0,015717 \mathrm{~g} \mathrm{NaOH})$ zur Neutralisation verlangte. $1 \mathrm{ccm} \mathrm{NaOH}=0,0168957 \mathrm{~g} \mathrm{CH}^{8} \mathrm{CO}=0,31763916 \mathrm{~g}=$ $35,97 \% \mathrm{CH}^{\mathrm{B}} \mathrm{CO}$-Gruppen.

Dieser Prozentgehalt an Acetylgruppen entspricht auf KHO bezogen $=46,84 \%$ KHO.

Gefunden für den Acetyljalapinsäureester in Prozenten:

$$
\begin{aligned}
& \mathrm{C}=53,30 \\
& \mathrm{H}=6,92 .
\end{aligned}
$$

\begin{tabular}{|c|c|c|c|c|c|}
\hline & & & & & \\
\hline & & 1. & 2. & 3. & 4. \\
\hline r Neutralisation & $\mathrm{n} \mathbf{K H C}$ & 2,93 & 2,76 & - & - \\
\hline Zur Verseifung & KHO & 47,8 & 50,47 & 50,52 & - \\
\hline & $\mathrm{CH}^{8} \mathrm{CO}$ & - & - & - & 35,97 . \\
\hline
\end{tabular}

Die früher ') bei der Untersuchung des Jalapins und der Jalapinsänre erhaltenen Werte in Betracht ziehend, kann der GlukosidoJalapinsäure = Jalapinsäure die Zusammensetzung $\mathrm{C}^{34} \mathrm{H}^{86} \mathrm{O}^{20}$ erteilt werden.

Die für die Jalapinsäure experimentell gefundenen Zahlen sind in Prozenten folgende:

$$
\begin{array}{cc}
\text { Gefunden: } & \text { Berechnet für } \mathrm{C}^{\mathrm{B}} \mathrm{H}^{60} \mathrm{O}^{20} ; \\
\mathrm{C}=51,54 & \mathbf{5 1 , 3 8} \\
\mathrm{H}=7,92 & \mathbf{8 , 3 1}
\end{array}
$$

Verbindungen von Kohlenhydraten mit den Oxysäuren sind von E. Fischer ${ }^{2}$ ) und L. Beensch ${ }^{8}$ ) dargestellt worden, die meisten derselben, welche bisher nicht krystallisierten und saure Reaktion haben, zerfallen bei der Hydrolyse in ihre Komponenten und besitzen den Charakter von Săureestern der betreffenden Kohlenhydrate.

Durch Eintritt von 10 Acetylgruppen in das Molekul der Jalapinsäure wurde ein Decaacetylester der Glukosido-Jalapinolsäure von der Zusammensetzung $\mathrm{C}^{84} \mathrm{H}^{56}\left(\mathrm{CH}^{8} \mathrm{CO}\right)^{10} \mathrm{O}^{20}$ dargestellt. Diese Verbindung erfordert in Prozenten:

$$
\begin{array}{rlr}
\text { Gefunden: } & \text { Berechnet für } \mathrm{C}^{\mathrm{A}} \mathrm{H}^{56}\left(\mathrm{CH}^{3} \mathrm{CO}\right)^{10} \mathrm{O}^{80}: \\
\mathrm{C}=53,30 & 53,37 \\
\mathrm{H}=6,92 & 7,09 \\
\mathrm{CH}^{8} \mathrm{CO}=35,97 & 35,42 .
\end{array}
$$

Der Vorgang der Hydrolyse durch Mineralsăuren kăme in der Weise za stande, dass durch Anfnahme von 1 Mol. Wasser die Jalapinsäure in 1 Mol. Jalapinolsäure und 3 Mol. einer Glykose zertällt: $\mathrm{C}^{84} \mathrm{H}^{86} \mathrm{O}^{20}+\mathrm{HO}=\mathrm{C}^{18} \mathrm{H}^{82} \mathrm{O}^{8}+3 \mathrm{C}^{6} \mathrm{H}^{18} \mathrm{O}^{6}$.

1) Zeitschrift des Oesterreichischen Apotheker-Vereins 1895, No. 18 u. ff.; Journal für praktische Chemie N. F. 1898, Bd. 57, pag. 448 u. ff.

2) Ber. d. d. chem. Ges. Bd. 26, 1893, pag. 2400.

3) Fischer und Beensch, Ber. d. d. chem. Ges. Bd. 27, 1894, pag. 2478. 
Bei dieser Reaktion werden, falls der dabei auftretenden Glykose die Zusammensetzung einer Hexose zuerteilt wird, $68 \%$ gebildet. Ermittelt wurden bei der Zugrundelegung des Reduktionswertes fur Traubenzucker volumetrisch $\mathbf{7 4 , 9 7 \%}$.

Das Jalapin kann als Glukosido-Jalapinolsäure angesehen werden, in welcher drei Methyläthylessigsäurereste eingetreten sind $-\mathrm{C}^{\mathbf{8 4}} \mathrm{H}^{\mathbf{6 8}}$ $\left(\mathrm{C}^{5} \mathrm{H}^{9} \mathrm{O}\right)^{8} \mathrm{O}^{20}$. Eine derartige Verbindung verlangt in Prozenten:

\begin{tabular}{ccc} 
Gefunden & \multicolumn{3}{c}{ Berechnet für } \\
für das Jalapin: & $\mathrm{C}^{84} \mathrm{H}^{83}\left(\mathrm{C}^{5} \mathrm{H}^{9} \mathrm{O}\right)^{8} \mathrm{O}^{20}$ & $\mathrm{C}^{84} \mathrm{H}^{82}\left(\mathrm{C}^{5} \mathrm{H}^{9} \mathrm{O}\right)^{4} \mathrm{O}^{\mathbf{2 0}}:$ \\
$\mathrm{C}=56,69$ & 56,21 & 57,34 \\
$\mathrm{H}=8,13$ & 9,56 & 8,67 \\
Zur Verseifung: & & \\
KHO $=21,68$ & - & - \\
$\mathrm{C}^{5} \mathrm{H}^{9} \mathrm{O}=32,9$ & 24,37 & $30,08$.
\end{tabular}

Das verbrauchte Kalihydrat stellt den Eintritt von 4 Methyläthylessigsäureresten in Aussicht, doch kommt der gefundene Kohlenstoffgehalt dem einen Tri-Methyläthylacetylester der Jalapinsäure nahe. Andererseits kann die gebundene Kalihydratmenge nicht allein als Mals für die Verseifung des Jalapins dienen, da wie nachgewiesen wurde, andere Oxysäuren auftreten, hauptsächlich $\alpha$-Methyl- $\beta$-Oxybuttersäure, die ihre Entstehung dem anwesenden Kalihydrat verdanken.

Mit Essigsäureanhydrid erhitzt, liefert das Jalapin den Pentaacetylester von der Zusammensetzung: $\mathrm{C}^{84} \mathrm{H}^{88}\left(\mathrm{C}^{5} \mathrm{H}^{9} \mathrm{O}\right)^{8}\left(\mathrm{CH}^{8} \mathrm{CO}\right)^{5} \mathrm{O}^{20}$. Mit Kalihydrat verseift liefert derselbe folgende Werte in Prozenten: Gefunden: $\quad$ Berechnet für $\mathrm{C}^{84} \mathrm{H}^{83}\left(\mathrm{C}^{5} \mathrm{H}^{8} \mathrm{O}\right)^{8}\left(\mathrm{CH}^{8} \mathrm{CO}\right)^{5} \mathrm{O}^{20}$ :

$$
\begin{aligned}
\mathrm{KHO} & =12,675 \\
\mathrm{CH}^{8} \mathrm{CO} & =17,37
\end{aligned}
$$$$
17,11 \% \text {. }
$$

Die Darmperistaltik anregende Wirkung der Convolvulaceenglykoside wird, wie es die Versuche von A. Scheuber ${ }^{1}$ ) wahrscheinlich gemacht haben, durch die in ihrer Konstitution vorkommende esterartige Bindung bedingt. In dem Acetyljalapin wäre somit eine drastischere Wirkung zu erwarten als es das Jalapin hervorzubringen vermag, ebenfalls wäre die Acetyljalapinsäure wirksamer als das A usgangsprodukt.

Dieses sind Fragen, deren wissenschaftliche Begründung vom pharmakologischen Standpunkte aus für die Therapie nicht ohne Nutzen sein kőnnte.

1) A. Scheuber, Ueber die Wirkung einiger Convolvulaceenharze, Diss. Jurjew. 1894. 